\title{
Magnetic field induced by elliptical instability in a rotating spheroid
}

\author{
By L. LACAZE, W. HERREMAN, M. LE BARS, S. LE DIZES and P. LE GAL \\ I.R.P.H.E., UMR 6594, CNRS - Université d'Aix-Marseille I \& II \\ Technopôle Château-Gombert, 49, rue F. Joliot-Curie, 13384 Marseille cédex 13
}

(Received 1 February 2006)

\begin{abstract}
The tidal or elliptical instability of rotating fluid flows is generated by the resonant interaction of inertial waves. In a slightly elliptically deformed rotating sphere, the most unstable linear mode is called the spin-over mode and is a solid body rotation versus an axis aligned with the maximum strain direction. In the non viscous case, this instability corresponds to the median moment of inertia instability of solid rotating bodies. This analogy is furthermore illustrated by an elliptical top experiment, which shows the expected inviscid heteroclinic behaviour. In geophysics, the elliptical instability may appear in the molten liquid cores of rotating planets, which are slightly deformed by tidal gravitational effects of close bodies. It may then participate to the general outer core dynamics and possibly to the geodynamo process. In this context, Kerswell and Malkus (Kerswell, R.R. and Malkus, W.V.R., Tidal instability as the source for Io's magnetic signature, Geophys. Res. Lett., 1998, 25, 603-606) showed that the puzzling magnetic field of the Jovian satellite Io may indeed be induced by the elliptically unstable motions of its liquid core that deflect Jupiter magnetic field. Our magnetohydrodynamics experiment is a toy-experiment of this geophysical situation and demonstrates for the first time the possibility of an induction of a magnetic field by the flow motions due to the elliptical instability. A full analytical calculation of the magnetic dipole induced by the spin-over is presented. Finally, exponential growths of this induced magnetic field in a slightly deformed rotating sphere filled with Galinstan liquid metal are measured for different rotating rates. Their growth rates compare well with theoretical predictions in the limit of a vanishing Lorentz force.
\end{abstract}

\section{The elliptical instability}

The elliptical instability (Kerswell, 2002) affects rotating flows when their cross-sectional streamlines are not exactly circular but slightly deformed into ellipses. It is known in particular that vortices are generically unstable when they are subject to a planar strain field. For instance, in a vortex pair, each vortex is subjected to the strain induced by the other vortex. The elliptical instability manifests in this system as axial undulations of the vortices (Leweke \& Williamson, 1998). This three-dimensional instability is also often invoked to explain the secondary three-dimensional instability of shear flows when several vortices have been created by Kelvin-Helmholtz type instabilities (Pierrehumbert, 1986). The instability is generated by a parametric resonance that couples together two inertial waves (Waleffe, 1990) and several experiments have already been devoted to the study of the elliptical instability in rotating deformed cylinders. In the very first experiments, rigid spinning cylinders with elliptical cross-sections or three-axial ellipsoids were considered (Gledzer et al., 1975). At the abrupt stop of the spinning body, e.g. during the transitory spin-down regime, axial undulations were briefly visualized before the complete stop of the fluid. To avoid this major disadvantage, Malkus (Malkus, 1989) invented the elastic rotating cylinder device where a transparent soft silicone cylinder is gently and permanently deformed during rotation. Malkus was able to visualise the appearance of the elliptical 


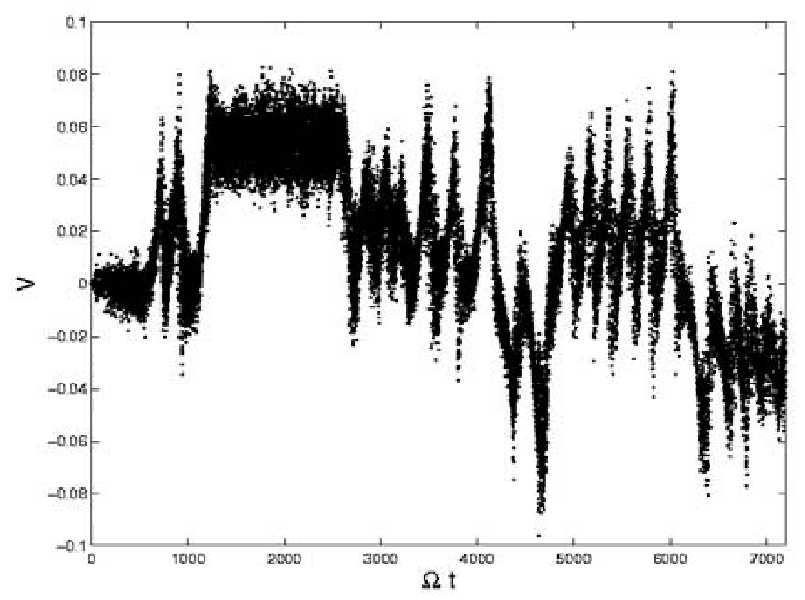

FiguRE 1. Doppler anemometry measurements of the radial velocity somewhere in the rotating deformed cylinder. Intermittent cycle of elliptical instability in the cylinder (where the phase of the unstable mode changes between two cycles) would imply a reversal of the magnetic field in MHD; one can also notice that the characteristic times of the dynamics range from the rotation period to thousands of rotation periods.

instability and for higher rotation rates, the complete breakdown of the flow through a kind of "resonant collapses" (MacEwan, 1970). This experimental arrangement was then extensively used by Eloy et al. (2003) who measured the linear and weakly non linear elliptical instability characteristics in the cylindrical geometry. In these experiments, stationary saturated regimes have been observed for high Ekman numbers. On the contrary, at low Ekman numbers, and similarly to the observations of Malkus (1989), intermittent regimes oscillating between turbulent and laminar flows take place. Our laser anemometry measures presented in figure 1 show this amazing intermittency where the phase of the instability can even change between two cycles (see the changes of sign of the radial velocity on figure 1).

Following these analyses, it was recently shown that, in a rotating deformed sphere (Lacaze et al., 2004), the most unstable mode of the elliptical instability is a rotation around an axis perpendicular to the container rotation axis. This mode, called the spin-over mode, can be visualised using the classical Kalliroscope technique as can be seen in Figure 2. The experimental container consists now in a hollow spherical cavity that is molded in a cylindrical elastic and transparent silicone gel cylinder. In order to cast this sphere, a ping pong ball was first inserted in a cylinder of liquid silicone that was then cured at a temperature of 50 Celsius. The ping-pong ball was finally dissolved by ethyl acetate injected inside the ball via $2 \mathrm{~mm}$ in diameter pipes. A hollow sphere molded in a transparent and deformable cylinder is finally obtained. The radius of this sphere is $21.75 \mathrm{~mm}$. The silicone cylinder is mounted on the vertical shaft of the device already used in the cylindrical case (Eloy et al., 2003) and is compressed between two vertical rollers. Note that these rollers are always in place; the device does not offer the possibility to move them in or out when rotation is started. The distance separating these rollers gives directly the elliptical deformation $\epsilon$ of the deformable sphere. The flow whose rotation axis is visualized by Kalliroscope flakes illuminated by a laser plane, consists as already said, in a solid body rotation with an axis given by the maximum strain direction. Because of the combination of the entrainment basic rotation around a vertical axis and of the spin-over mode, the axis of rotation of the fluid in the core of the flow is tilted as can be seen in Figure 2. The S shape comes from the presence of viscous Ekman layers that arise from the differential rotation between the fluid and the container. As shown in Lacaze et al. (2004) other modes of the elliptical instability can also become linearly unstable. However, the spin-over mode growth rate is always the largest at threshold and apart from 


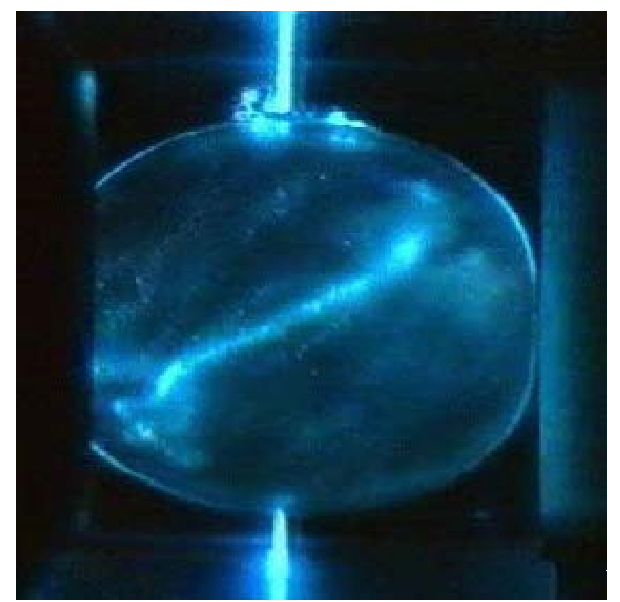

FIGURE 2. Kalliroscope visualisation of the spin-over mode of the elliptical instability in a rotating sphere filled with water. The observation plane is parallel to the spin-over axis. The sphere rotates around a vertical axis while it is slightly compressed by two rollers. The tilted axis of flow rotation is clearly visible by the use of Kalliroscope flakes. This inclination of the flow rotation axis comes from the combination of the main rotation imposed by the container and by the unstable spin over mode which is a solid body rotation around an horizontal axis.

high $\epsilon$, higher modes are not expected to be selected by linear processes. Moreover, as it was the case in the cylindrical geometry, at low Ekman numbers $\mathrm{E}\left(E=\nu / \Omega R^{2}\right.$, where $\nu$ is the fluid viscosity, $\Omega$ the rotation rate and $\mathrm{R}$ the radius of the container), the spin-over mode is destabilised and an intermittent regime takes place. To complete the study, a solid core was finally placed in the center of the sphere: experimental and theoretical analyses then showed the persistence of the spin-over in the spherical shell with a typical growth rate $\sigma$ given by

$$
\sigma=\left(\epsilon \sigma_{N V}-E^{1 / 2} \sigma_{V}\right) \Omega
$$

where $\epsilon$ is the ellipticity, $\sigma_{N V}$ and $\sigma_{V}$ are functions of the inner to outer spheres ratio $\eta\left(\sigma_{N V}=0.5\right.$ and $\sigma_{V}=2.62$ for $\eta=0, \sigma_{N V}=0.5$ and $\sigma_{V}=1.8$ for $\eta=1 / 3$ ) (see Lacaze et al., 2005).

\section{The elliptical top: a model for the the spin-over mode of the inviscid flow}

In fact, the mechanism of appearance of this rotation in the core of the fluid sphere (the spin-over), is nothing else than the instability of rotation of a solid around its median axis of inertia. This phenomenon is often illustrated in physics classes by the throw of a spinning box of matches which deviates from the rotation initially imposed around its median axis of inertia. In a more quantitative way, one can show that the non viscous and nonlinear problem of the solid rotation of the fluid in the laboratory frame of reference is completely equivalent to the problem of the stability of the rotation of a solid ellipsoid in the rotating reference frame. In both cases, the equations of motions are the Euler equations and read:

$$
\begin{gathered}
(2-\epsilon) \dot{\Omega_{1}}=-\epsilon\left(1+\Omega_{3}\right) \Omega_{2}, \\
(2+\epsilon) \dot{\Omega_{2}}=-\epsilon\left(1+\Omega_{3}\right) \Omega_{1}, \\
\dot{\Omega_{3}}=\epsilon \Omega_{1} \Omega_{2},
\end{gathered}
$$




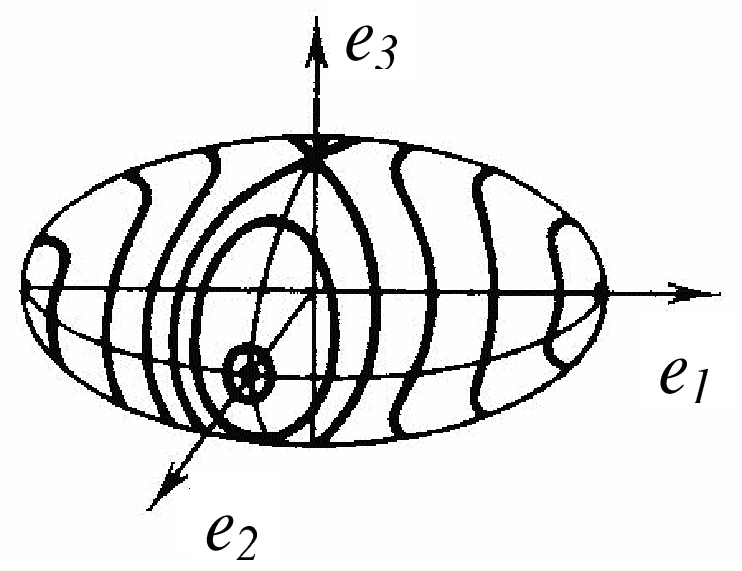

Figure 3. The heteroclinic trajectories, which pass by the poles of the ellipsoid, illustrate the instability of the rotation of a solid versus its median axis of inertia.

where the $\Omega_{i}$ are the projections on the three principal axes of the instantaneous rotation vector disturbance and where $\Omega$ was normalised to one. The dot represents time derivative and the median axis of inertia is the axis $e_{3}$ which is the unstable axis of rotation. The dilation and the compression of the ellipsoid were carried out according to the first and the second axis with an amplitude $\epsilon$. The solutions of this system of equations are heteroclinic trajectories which pass near the unstable fixed points of the poles. These trajectories are illustrated in figure 3.

In order to experimentally illustrate the dynamics of such a spinning solid ellipsoid that would imitate the motion of an inviscid fluid contained in a rotating sphere undergoing the effects of a tide, a spinning top having three different moments of inertia was realised. The two poles of its median axis of inertia are marked by two colour stickers which are used to observe the axis of rotation during spinning. Figure 4 shows the two first cycles of the heteroclinic dynamics as described previously: we can observe an alternation of the poles marked by the coloured stickers. Of course, even if friction in this case of solid dynamics is much less than in the case of a fluid (only one contact point with the table), the spinning top ends up deviating from this heteroclinic trajectory and is attracted by the rotation around one of the axes of greater or smaller moment of inertia: both centres, seen in figure 3 are now transformed into two stable focus because of energy dissipation.

In a similar way, the motions in the viscous fluid ellipsoid, due to the elliptical instability, do not describe the inviscid heteroclinic cycles, at least for the Ekman numbers considered in this study. The angle of the tilted axis of rotation saturates after a slight overshoot. On the other hand, it is relatively easy to follow the angle of this axis as a function of time and deduce the instability growth rate for various Ekman numbers. A theoretical analysis which takes into account the nonlinear and viscous effects was also carried out (Lacaze et al., 2004). The equations of motion are then modified in the following way:

$$
\begin{gathered}
\dot{\Omega_{1}}=-\frac{\epsilon}{(2-\epsilon)}\left(1+\Omega_{3}\right) \Omega_{2}+\nu_{S O} \Omega_{1}, \\
\dot{\Omega_{2}}=-\frac{\epsilon}{(2-\epsilon)}\left(1+\Omega_{3}\right) \Omega_{1}+\nu_{S O} \Omega_{2}, \\
\dot{\Omega}_{3}=\epsilon \Omega_{1} \Omega_{2}+\nu_{E C} \Omega_{1}+\nu_{N L}\left(\Omega_{1}^{2}+\Omega_{2}^{2}\right),
\end{gathered}
$$

where the different coefficients have been calculated following Greenspan (1968): $\nu_{S O}=-2.62 E^{1 / 2}$ (for the linear damping rate of the spin-over), $\nu_{E C}=-2.85 E^{1 / 2}$ (for the linear damping rate of the axial rotation) and $\nu_{N L}=1.42 E^{1 / 2}$ (for the damping in the boundary layer due to the non linear 


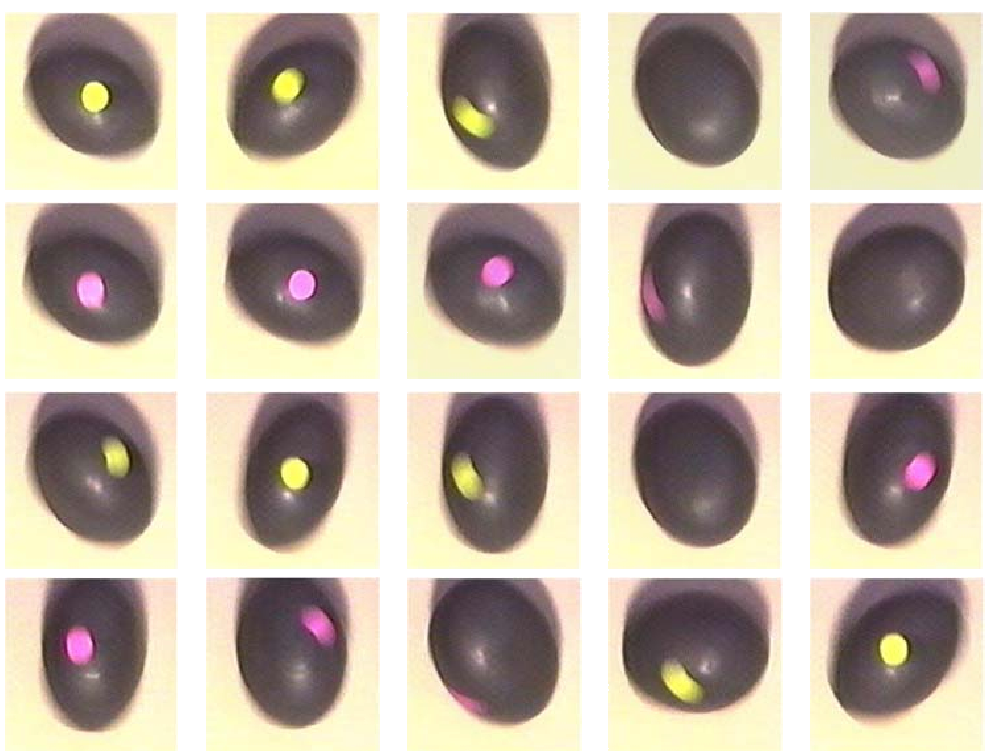

FIgURE 4. Series of chronological images showing two heteroclinic cycles of the axis of rotation of the elliptical spinning top.

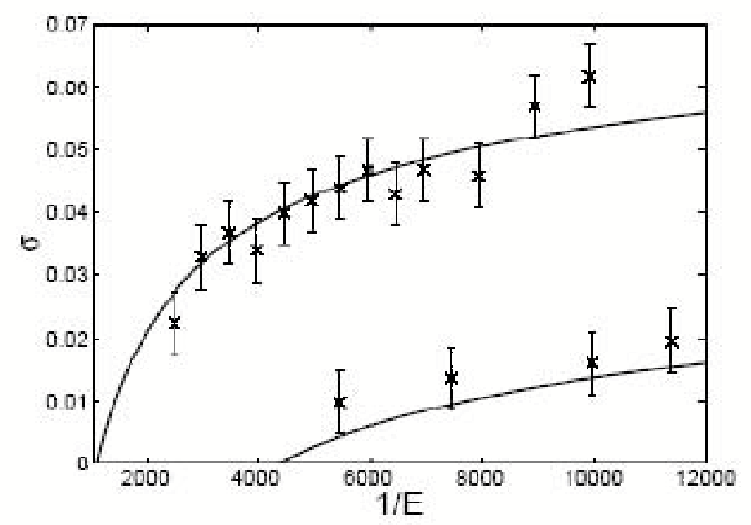

FigURE 5. Comparison between the linear analysis of elliptical instability in the sphere and experimental measurements of the growth rates $\sigma$ of the spin-over for two cases of ellipticity $(\epsilon=0.08$ and 0.16$)$.

interaction of the spin-over with itself). The viscous and nonlinear dynamics confirms the selection of the spin-over mode whose growth rate $\sigma$ have been reproduced from Lacaze et al. (2004) in figure 5. As can be seen, a good agreement is obtained between theory and experiment. Note that the extrapolation presented in figure 6 is obtained from these analyses.

\section{The elliptical instability in geophysics}

If up to now, most of the studies about the elliptical instability concern shear flows and vortices in fluids, another fundamental interest in the study of elliptical instabilities is related to the understanding of geophysical and astrophysical flows. In particular, despite a wealth of studies, planetary 
magnetic fields remain a challenging interdisciplinary research topic at the international level (see for example the recent review of Busse, 2000). The main point is to understand how planets - and especially the Earth - can generate and maintain a magnetic field over durations of time much longer than the typical magnetic diffusion time. The commonly accepted answer is that such fields are due to motions in an electrically conducting fluid within the planets. But the appropriate energy source as well as the organisation of these motions remain (among others) open questions. On Earth, the prevalent and most reasonable hypothesis is that the present dynamo is powered by thermo-chemical convection within the conducting core driven by the crystallisation of the inner core: multiple studies have thus been performed (see for instance the review of Busse (2002) and references therein). Following the Earth's example, it is commonly and tacitly assumed that the generation of a magnetic field in other planetary cores is also due to convection. However, other mechanisms have been suggested as for instance inertial instabilities due to precession and/or tides (e.g. Vanyo et al., 1995, Kerswell and Malkus, 1998, Noir et al., 2001 and Tilgner, 2005), which could strongly influence the dynamics of the Earth's liquid core, and even generate or induce a magnetic field in other planets or in the early Earth, i.e. before the beginning of the inner core crystallisation one billion years ago (Labrosse et al., 2001). Actually, on no account planetary dynamo systematically means core's convection. For instance, the recent flybys of the Galilean satellites by the Galileo mission have brought the surprising conclusions that Io may have an intrinsic magnetic field, and that Ganymede has a permanent dipole due to a recent or present dynamo (Kivelson et al., 1996a,b). In both cases however, the traditional convective scenario derived from the Earth's case is not entirely satisfying: convection is impossible in Io's core because of the steadily tidal heating of its mantle (Wienbruch and spohn, 1995), and sustaining convection over billions of years is very surprising in Ganymede's core because of its small size (Stevenson, 2003). Taking into account the importance of tides in Galilean satellites (deformation of several hundred meters in Io for instance), Kerswell and Malkus (1998) have thus suggested that these magnetic fields could be due to tidal instabilities.

Inertial instabilities due to precession and tides come from the same physical mechanism. As in laboratory rotating flows, precession or tides in rotating planetary liquid cores can also excites pairs of inertial waves selected according to the geometry of the container and of the forcing. The linear theory then shows a divergence of the amplitude of the selected modes, and the structure of the flow observed experimentally changes radically. Because of this parametric resonance, inertial effects can be of primary importance even in flows at (estimated) low Rossby numbers, as for example in planets. Then, this parametric resonance would radically change the usual vision of motions in planetary cores, for instance invalidating the Proudman-Taylor constrain, often called upon on Earth.

In this context, the studies in the sphere (Lacaze et al., 2004) and in the spherical shell (Lacaze et al., 2005) are especially interesting from a geophysical point of view. Indeed, we can now quantitatively estimate the presence of an elliptical instability in a planetary core given by $\sigma>0$ (see equation 5): as shown in figure 6, an elliptical instability is proved on Io and possible on Earth (see also Kerswell, 1994). Besides, we can also quantitatively demonstrate that the typical timescale of the inertial instabilities (given by $1 / \sigma$ ) is much larger than the rotation rate (see also Aldridge et al., 1997). Finally, the intermittency cycles and the phase reversals observed in the hydrodynamics at low Ekman numbers (see figure 1) could translate into magnetic field excursions and reversals in planets. All these points thus suggest that tidal instabilities could create realistic magnetic fields at the planetary scale.

However, the previous conclusions are only based on hydrodynamics ground. A systematic study of the magnetohydrodynamics of the inertial instabilities is now necessary. Regarding geophysical applications, two different problems arise: first, from a purely MHD point of view, one can wonder what the characteristics of the induced field are (intensity, dipolar/quadripolar, orientation) and how the hydrodynamical instability is in turn modified by the induced field; then, one can wonder whether tidal instabilities can indeed initiate and power a dynamo in the parameters range relevant to the planets, as recently demonstrated numerically by Tilgner (2005) in the closely related case of precession. 


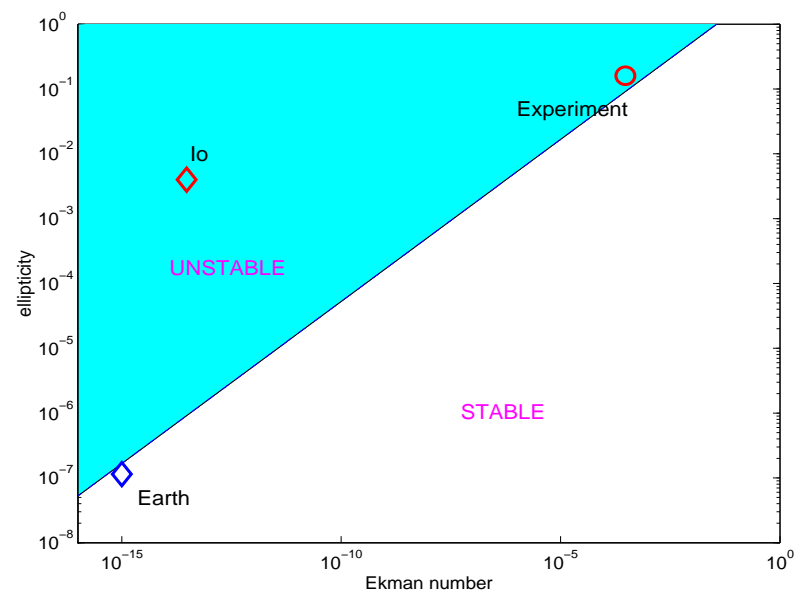

Figure 6. Stability of a flow in a sphere in the presence of an elliptical deformation, depending on the Ekman number and on the imposed ellipticity. Data for Io come from Kerswell and Malkus (1998) and are derived from Anderson et al. (1996). The straight line is given by $\sigma=0$ (see equation 1.1)

In the present paper, our main objective is to answer the first question, which is interesting on its own, but can also be considered as a first step toward the second one. We thus aim at systematically quantifying the characteristics of the magnetic field induced by tidal instabilities in various configurations. Theoretical and experimental studies have been performed concurrently and provide complementary results. In the first part of section 4, analytical results are presented that precisely determine the influence of an imposed magnetic field on a simplified spin-over mode (i.e. no Ekman layers are taken into account): the shape of the induced magnetic field and its intensity are determined over very large parameters range, especially regarding the magnetic Reynolds number. Experimental results are described in the second part of section 4: measurements of the magnetic field induced by the elliptical instabilities are presented for magnetic Reynolds numbers around $10^{-2}$.

\section{Magnetic field induction by elliptical instability.}

We have seen that the spin-over mode is spontaneously generated by the elliptical instability in a slightly deformed rotating spheroid of non-magnetic fluid. This simple hydrodynamic eigenmode of the uniformly rotating sphere, is unaffected when dealing with liquid metals in the limit of small magnetic fields: magnetohydrodynamics reduces to hydrodynamics and the induction equation when the Elsasser number is small. A liquid metal analogue of the former experiment in presence of a small external magnetic field should thus be able to excite the same spin-over mode. However, this time, it would be accompanied by an induced magnetic field, resulting from the interaction of the flow with the imposed field. This magnetic signature would also be visible from the exterior where we aim to detect it. In this section, our first goal is to provide an analytic description of the magnetic field induced by the spin-over mode inside and outside the sphere. Our second goal is to provide experimental measurements of this induced magnetic field.

In the experimental configuration, a small homogeneous vertical magnetic field of 14 Gauss is created by two Helmholtz coils (see figure 7). The imposed magnetic field $\mathbf{H}_{0}=H_{0} \mathbf{e}_{z}$ is parallel to the axis of rotation of the sphere. The sphere rotates at the angular rotation $\Omega$ and is filled with a GalliumIndium-Tin eutectic (Galinstan). This metal has the advantage over Gallium to be liquid at room temperature. Its density is $\rho=6.4410^{3} \mathrm{~kg} \mathrm{~m} \mathrm{~m}^{-3}$, its kinematic viscosity is $\nu=0.9310^{-6} \mathrm{~m}^{2} \mathrm{~s}^{-1}$ and its magnetic diffusivity is $\nu_{m}=0.36 \mathrm{~m}^{2} \mathrm{~s}^{-1}$. 


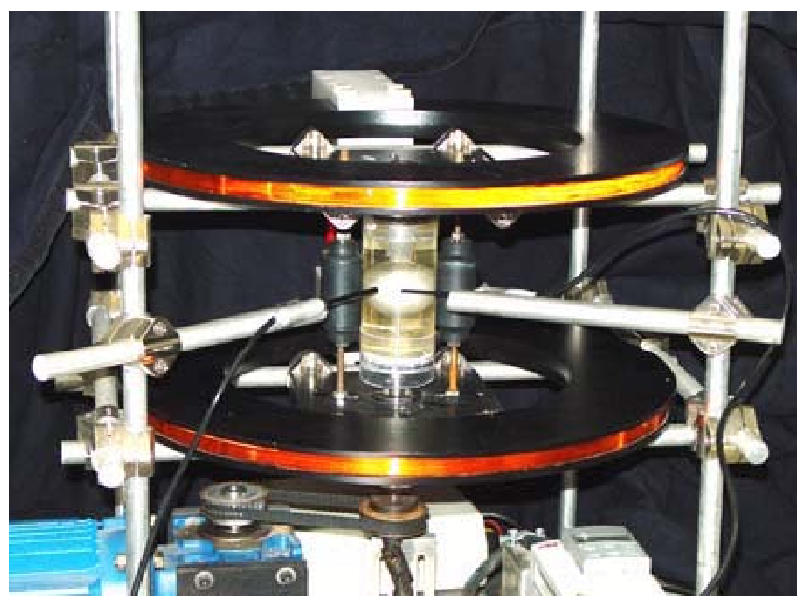

FiguRE 7. Experimental set up with the pair of Helmholtz coils surrounding the rotating sphere and the Hall effect probes.

A small elliptical deformation is applied to the sphere by using the rollers in order to excite the spin-over mode. In the experiment, the ellipticity of the streamline is approximatively $\epsilon=0.095$. If we neglect the viscous Ekman layers, the spin-over mode corresponds to a solid body rotation of angular rotation $\Omega_{S O}$ around a horizontal axis, say $O x$. The total flow in presence of this mode is thus approximatively a solid-body rotation around an axis parallel to $\Omega_{S O} \mathbf{e}_{x}+\Omega \mathbf{e}_{z}$. The associated velocity field is noted $\mathbf{U}_{T}$.

The magnetic field induced by this flow in presence of the imposed field $\mathbf{H}_{0}$ can be calculated exactly. If we write the total magnetic field (inside and outside the sphere) as $\mathbf{H}_{T}=\mathbf{H}_{0}+\mathbf{H}$, the induced field $\mathbf{H}$ is found to satisfy, in a fixed frame, the equations:

In the sphere $(r<1)$ :

$$
\begin{array}{r}
\frac{\partial \mathbf{H}_{i}}{\partial t}+\nabla \times\left(\mathbf{U}_{T} \times \mathbf{H}_{i}\right)-\frac{1}{R e_{m}} \nabla^{2} \mathbf{H}_{i}=-\nabla \times\left(\mathbf{U}_{T} \times \mathbf{H}_{0}\right), \\
\nabla \cdot \mathbf{H}_{i}=0 ;
\end{array}
$$

Outside the sphere $(r>1)$

$$
\begin{array}{r}
\mathbf{H}_{e}=\nabla \Psi \\
\nabla^{2} \Psi=0 .
\end{array}
$$

The indexes $i$ and $e$ refer to internal and external fields respectively. The magnetic fields have been non-dimensionalized by $H_{0}$ (such that $\mathbf{H}_{0}=\mathbf{e}_{z}$ ), spatial variables by the sphere radius $R$, and time by $\Omega^{-1}$. The magnetic Reynolds number is defined by

$$
R e_{m}=\frac{\Omega R^{2}}{\nu_{m}},
$$

where $\nu_{m}=\left(\sigma_{m} \mu_{m}\right)^{-1}$ with $\sigma_{m}$ the fluid conductivity, and $\mu_{m}$ its magnetic permeability.

Inside the sphere, the dynamics of the magnetic field is controlled by the induction equation, whereas the field is the gradient of a scalar potential field $\Psi$ outside the sphere (no source of magnetic field). Magnetic fields are always solenoidal as prescribed by (4.2) and (4.4). In addition, the total magnetic field has to be continuous at the spherical boundary, a consequence of $\mu \simeq \mu_{0}$ and the usual boundary conditions for the magnetic induction and field.

To obtain the induction field, it is useful to consider the problem for the full magnetic field in the rotating frame where the fluid is at rest. In this frame, the induction equation inside the sphere reduces 
a)

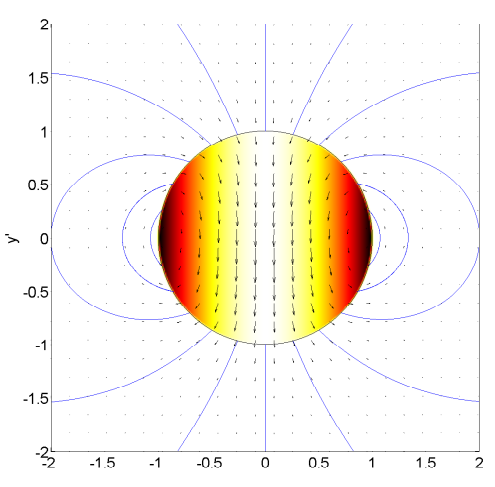

b)

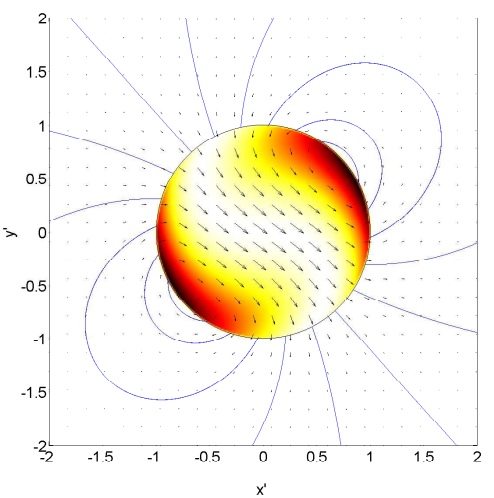

c)

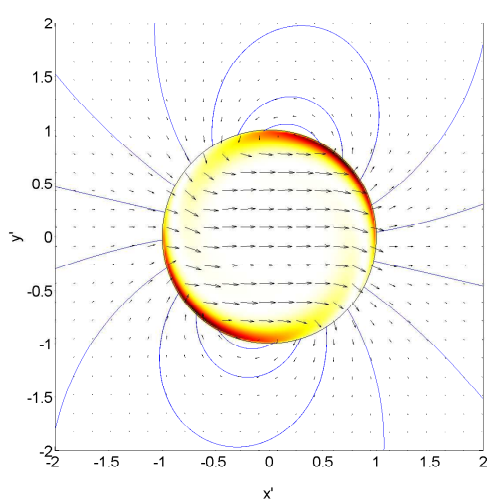

Figure 8. Arrows represent the induced magnetic field in the (tilted) equatorial plane $\left(\theta^{\prime}=\pi / 2\right)$. Field lines outside the sphere are solid lines. Joule dissipation $\left|\nabla \times \mathbf{H}^{\prime}\right|^{2}$ inside the sphere is indicated by the gray scale. (a) $R e_{m}^{\prime}=0.01$, (b) $R e_{m}^{\prime}=10$, (c) $R e_{m}^{\prime}=100$. Note that the field is perpendicular to the spin-over axis at low $R e_{m}^{\prime}$ but tends to be parallel at higher $R e_{m}^{\prime}$.

to a diffusion equation. Outside the sphere, the equations are unchanged, but the uniform external field $\mathbf{H}_{0}$ becomes oscillating with a frequency $\sqrt{1+\Omega_{S O}^{2}}$ (with our normalisation). This oscillating behaviour prescribes the time-dependency of the field inside the sphere. The diffusion equation then becomes a Helmholtz equation which can be solved in the spherical geometry using classical techniques (see Morse and Feshbach, 1953). The idea is then to return to a fixed frame in which the induced field is stationary. The expression can be simplified by considering a tilted frame, such that its vertical axis $\mathbf{e}^{\prime}{ }_{z}$ is oriented along with the rotation axis of the total flow (see figure 10). In this fixed frame, the imposed magnetic field $\mathbf{H}_{0}$ can be written as $\mathbf{H}_{0}=-\sin \theta_{0} \mathbf{e}^{\prime}{ }_{x}+\cos \theta_{0} \mathbf{e}^{\prime}{ }_{z}$ where the inclination angle $\theta_{0}$ is connected to the normalised spin-over rotation rate by

$$
\sin \theta_{0}=\frac{\Omega_{S O}}{\left(1+\Omega_{S O}^{2}\right)^{1 / 2}}
$$

If we express the cylindrical components $\left(H_{\rho}^{\prime}, H_{\phi}^{\prime}, H_{z}^{\prime}\right)$ of the induced magnetic field in terms of spherical variables $\left(r^{\prime}, \theta^{\prime}, \phi^{\prime}\right)$, we obtain an expression composed of two different parts:

$$
\mathbf{H}_{i}^{\prime}\left(r^{\prime}, \theta^{\prime}, \phi^{\prime}\right)=\sin \theta_{0}\left|A\left(r^{\prime}\right)\right|\left(\begin{array}{c}
\cos \left[\phi^{\prime}+\chi_{A}\left(r^{\prime}\right)\right] \\
\sin \left[\phi^{\prime}+\chi_{A}\left(r^{\prime}\right)\right] \\
0
\end{array}\right)
$$




$$
\begin{gathered}
+\sin \theta_{0}\left|B\left(r^{\prime}\right)\right|\left(\begin{array}{c}
\frac{1}{4}\left(3 \cos 2 \theta^{\prime}-1\right) \cos \left(\phi^{\prime}+\chi_{B}\left(r^{\prime}\right)\right) \\
-\frac{1}{2} \sin \left(\phi^{\prime}+\chi_{B}\left(r^{\prime}\right)\right) \\
-\frac{3}{4}\left(\sin 2 \theta^{\prime}\right) \cos \left(\phi^{\prime}+\chi_{B}\left(r^{\prime}\right)\right)
\end{array}\right), \\
\mathbf{H}_{e}^{\prime}\left(r^{\prime}, \theta^{\prime}, \phi^{\prime}\right)=\mathbf{H}_{i}^{\prime}\left(1, \theta^{\prime}, \phi^{\prime}\right) r^{\prime-3},
\end{gathered}
$$

where $|A|,|B|, \chi_{A}$ and $\chi_{B}$ are defined by

$$
\begin{aligned}
& A\left(r^{\prime}\right)=\left|A\left(r^{\prime}\right)\right| e^{i \chi_{A}\left(r^{\prime}\right)}=1-\frac{j_{0}\left(k r^{\prime}\right)}{j_{0}(k)}, \\
& B\left(r^{\prime}\right)=\left|B\left(r^{\prime}\right)\right| e^{i \chi_{B}\left(r^{\prime}\right)}=\frac{j_{2}\left(k r^{\prime}\right)}{j_{0}(k)} .
\end{aligned}
$$

In these formulas, $j_{0}$ and $j_{2}$ are spherical Bessel functions and the parameter $k$ is given by $k=$ $\sqrt{-i R e_{m}}\left(1+\Omega_{S O}^{2}\right)^{1 / 4}$. With these expressions, one can clearly see that the $A$-part of the solution only exists inside the sphere and disappears at $r^{\prime}=1$, and that it has no vertical component. It can be interpreted as the field needed to compensate the transverse part of the imposed field in the tilted frame. The $B$-part exhibits almost opposite behaviour since it vanishes at the origin, becomes more and more important as we approach the border, and extends to the outside. This part of the solution is necessary since the A-part by its own cannot be matched to the external potential field. The structure of the magnetic field is a dipole outside the sphere. However, inside the sphere, the field lines are deformed by the rotation of the fluid. The magnetic Reynolds number appears only via a renormalised magnetic Reynolds number $R e_{m}^{\prime}=R e_{m} \sqrt{1+\Omega_{S O}^{2}}$, which takes into account the rotation of the spin-over mode.

A few symmetries can be pointed out from the above expressions. The equatorial plane $\theta^{\prime}=\pi / 2$ is a mirror-symmetry plane for the internal as well as the external field. In this plane, $\mathbf{H}^{\prime}$ has no vertical $\mathbf{e}^{\prime}{ }_{z}$ component. Field lines in this plane are plotted in figure 8 for three different values of $R e_{m}^{\prime}$.

The intensity of the Joule dissipation $\left|\nabla \times \mathbf{H}^{\prime}\right|^{2}$ within the liquid metal is also indicated by the gray scale. For small $R e_{m}^{\prime}$, the dipole field points in the $-\mathbf{e}^{\prime}{ }_{y}$ direction, both inside and outside the sphere. Field amplitudes are small, and dissipation seems to be rather well distributed over the entire circle. For moderate $R e_{m}^{\prime},\left(R e_{m}^{\prime}=O(1)\right)$, we observe that the external field starts to change its orientation toward the x'-axis. Inside the sphere, we can see that dissipation begins to be enhanced close to the boundary, and field amplitudes increase. For higher $R e_{m}^{\prime}$, the external field is a dipole field aligned with the x'-axis. Inside the sphere, dissipation concentrates in a fine region close to the border, and a rather uniform magnetic field establishes in the $\mathrm{x}^{\prime}$-direction. The field magnitude never ceases to grow with increasing $R e_{m}^{\prime}$ but never becomes bigger than $H_{0} \Omega_{S O} / \Omega$ (see figure 9 -a). The tilting of the external dipole as $R e_{m}^{\prime}$ increases can be understood from the full field expression. Indeed, the external field possesses a symmetry axis along the direction $\phi^{\prime}=-\chi_{B}(1)$ in the equatorial plane $\theta^{\prime}=\pi / 2$. The evolution of $|B(1)|$ and $\chi_{B}(1)$ with respect to $R e_{m}^{\prime}$ is plotted in figure 9 .

It is also interesting to discuss the effect of variation of $\Omega_{S O}$ on the magnetic field. The amplitude of the spin-over mode appears in the rescaled magnetic Reynolds number and in the definition of the angle $\theta_{0}$. Increasing $\Omega_{S O}$ is therefore expected to have two effects: it increases the tilting of the frame, and increases the rescaled magnetic Reynolds number which has been shown also to tilt the dipole axis of the external field. We now consider two asymptotic limits $R e_{m} \rightarrow \infty$ and $R e_{m} \rightarrow 0$ for which the expressions for the magnetic field become particularly simple. The first limit $\left(R e_{m} \rightarrow \infty\right)$ corresponds to ideal MHD. This limit permits to show how magnetic boundary layers develop. The second limit $\left(R e_{m} \rightarrow 0\right)$ is close to the experimental conditions.

For $R e_{m} \rightarrow \infty$, we find

$$
A\left(r^{\prime}\right) \sim 1-\exp \left(-\frac{(1-i)}{\sqrt{2}} \operatorname{Re}_{m}^{1 / 2}\left(1+\Omega_{S O}^{2}\right)^{1 / 4}\left(1-r^{\prime}\right)\right)
$$


a)

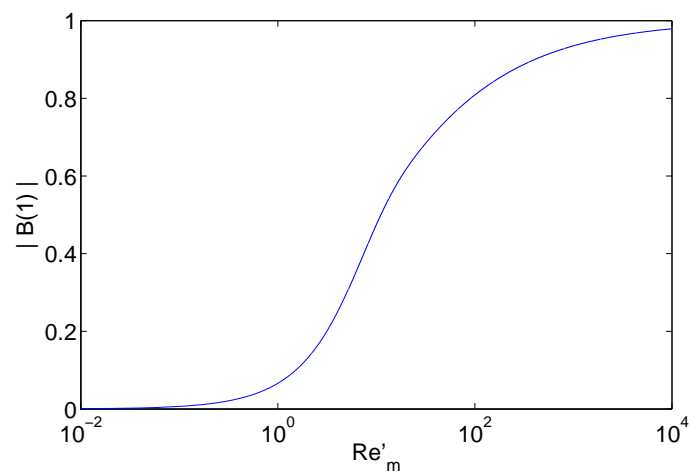

b)

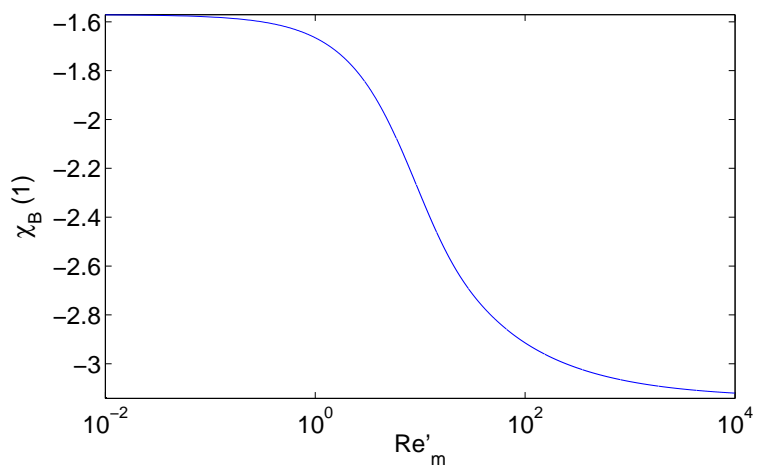

Figure 9. Variation of the dipole magnitude $|B(1)|$ (a) and orientation angle $\chi_{B}(1)$ (b) with respect to $R e_{m}^{\prime}$.

$$
B\left(r^{\prime}\right) \sim-\exp \left(-\frac{(1-i)}{\sqrt{2}} R e_{m}^{1 / 2}\left(1+\Omega_{S O}^{2}\right)^{1 / 4}\left(1-r^{\prime}\right)\right) .
$$

From these expressions, we see that within the sphere, for large $R e_{m}, A$ is constant and $B$ is exponentially small. The resulting magnetic field $\mathbf{H}_{i}$ is thus uniform and oriented with the spin-over rotation axis, which is in agreement with figure $8(\mathrm{c})$. It is only in a fine region of order $R e_{m}^{-1 / 2}$ near the inner boundary of the sphere $\left(r^{\prime} \approx 1\right)$, that $A$ and $B$ vary. This magnetic boundary layer permits to match the uniform magnetic field with a decreasing potential field outside the sphere. It is worth mentioning that the $B$-part of the field, which is generated in the boundary layer, remains present outside the sphere far from the boundary. This demonstrates the non-trivial nature of the large magnetic Reynolds number limit.

For $R e_{m} \rightarrow 0$, we obtain

$$
\begin{gathered}
A\left(r^{\prime}\right) \sim i R e_{m} \sqrt{1+\Omega_{S O}^{2} \frac{\left(1-r^{\prime 2}\right)}{10}}+O\left(R e_{m}^{2}\right), \\
B\left(r^{\prime}\right) \sim-i R e_{m} \sqrt{1+\Omega_{S O}^{2}} \frac{r^{\prime 2}}{35}+O\left(R e_{m}^{2}\right),
\end{gathered}
$$

which shows that the induced magnetic field is small. Moreover, as both A and B are purely imaginary at leading order, both external and internal fields have now an axis of symmetry in the $\mathbf{e}_{y}^{\prime}$ direction. This direction also corresponds to $\mathbf{e}_{y}$. The structure of the induced magnetic field can be understood in this limit as a torsion of the imposed magnetic field lines by the spin-over mode as schematically illustrated in figure 10. This effect corresponds to the so-called $\Omega$-effect in the MHD literature. Indeed, along the $O y$ line, the external field possesses a particularly simple expression:

$$
\mathbf{H}_{e}=-\frac{\Omega_{S O} R e_{m}}{35 r^{3}} \mathbf{e}_{y} .
$$

This expression is valid whatever the amplitude of the spin-over mode (as long as $R e_{m}^{\prime}$ remains small). In the experiment, the measurements are performed outside the sphere along the line $O y$. The magnetic Reynolds number of the flow is of the order of $10^{-2}$ and the Elsasser number which compares the Lorenz force with inertial forces is of order $10^{-4}$. The magnetic field is therefore not expected to modify the hydrodynamic evolution and (4.14) can be used for the expression of the magnetic field at the probe. What is important to note is that the induced magnetic field at the probe is proportional to $\Omega_{S O}$ whatever the amplitude of the spin-over mode. The slow evolution of spin-over mode at instability threshold, in particular its exponential growth and saturation, is therefore expected to be captured by the magnetic field measurement. This is demonstrated in figure 11a) where the exponential growth of the induced field is recorded by a Hall effect probe positioned outside the sphere, in the equatorial 


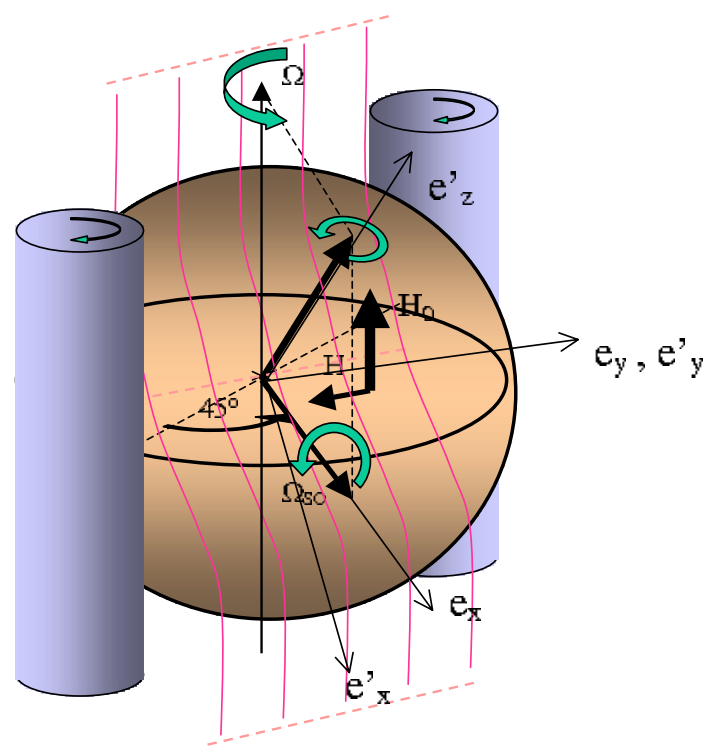

FIGURE 10. Schematic representation of the distorted magnetic field lines associated with the $\Omega$ effect generated by the spin-over mode of the elliptical instability in the rotating deformed sphere.

plane and aligned with the $O y$ axis. The vertical imposed magnetic field had a value of 14 Gauss at the external position of the probe and the radial induced field reaches 6 mGauss. This compares well with formula 4.14, which gives an estimated value of 4.2 mGauss, taking $\Omega_{S O}=1, R e_{m}=10^{-2}$ and $r=1$. Different growth rates $\sigma$ can thus be measured when the rotation rate $\Omega$ (and thus the Ekman number) of the flow is changed. Figure 11b) presents the evolution of $\sigma$ with the Ekman number. The solid curve is drawn from the theory where the Lorenz force was not taken into account and for an ellipticity value of $\epsilon=0.095$. Note that there is no free parameter in equation 1.1 and that the agreement between the theory and the experiment is very good. The error bars are estimated from the exponential fit calculation of the growth curves. Note that their size is of the same order of magnitude than those obtained from the visualization method (see Figure 1.1). In this case, the errors come from the difficulty we had to determine the position of the axis of rotation. In the present magnetic field growth rates measurement, some strong oscillations perturb the induction signal as can be seen on figure 11-a). These magnetic perturbations cause the major lack of accuracy in the growth rates determination. They are induced when starting the electric motor of our device and they appear to be difficult to avoid in the present set-up where the imposed field and consequently the induced field are very weak. We expect to improve the signal to noise ratio by using stronger magnetic fields in the future.

\section{Conclusion.}

We have presented a theoretical and experimental study of the hydrodynamics and magnetohydrodynamics of the elliptical or tidal instability in a spherical geometry. This geometry is of great interest for geophysical applications. Experiments as well as a theoretical analysis have been performed in a deformable rotating sphere: we have characterized the growth of the most unstable mode called the spin-over, which forces the fluid to rotate along an axis perpendicular to the axis of entrainment, and gives a "S" shape to the axis of rotation of the fluid. We have then presented an analogy of this fluid flow instability with the median moment of inertia instability of spinning solid bodies in the inviscid 
a)
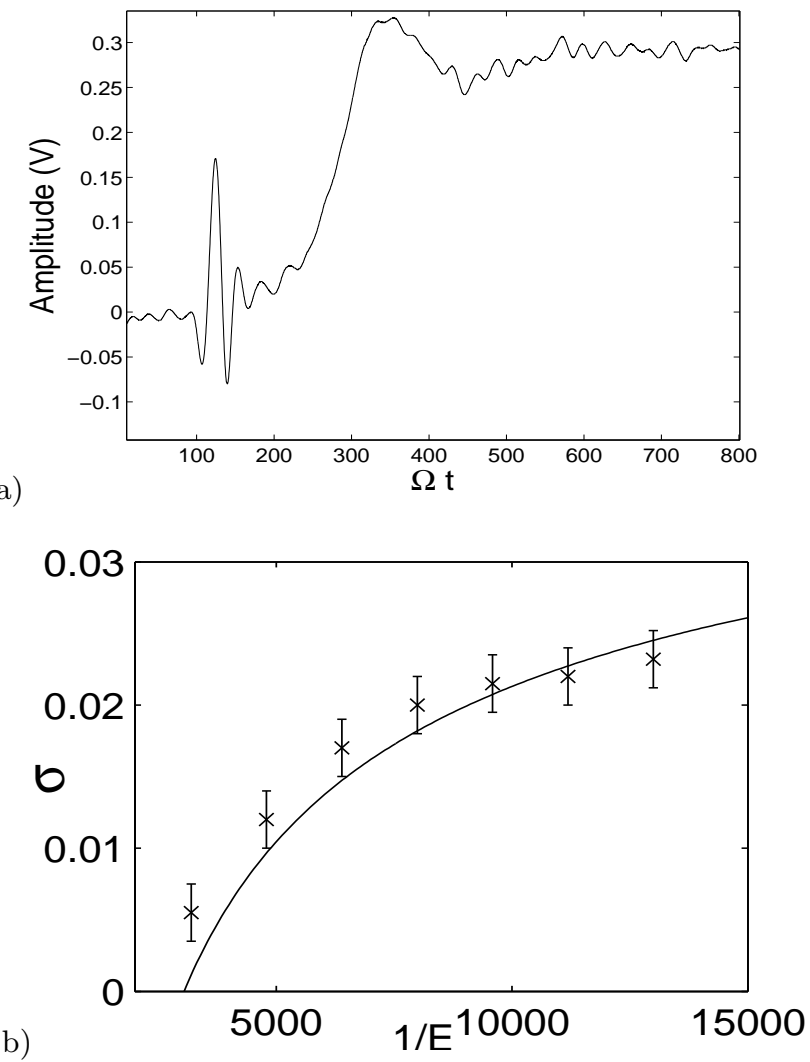

Figure 11. a) Temporal exponential growth of the induced magnetic field ( $50 \mathrm{mV} / \mathrm{mGauss})$. The vertical imposed magnetic field had a value of 14 Gauss at the external position of the probe and the radial induced field reaches 6 mGauss. The oscillations for $100<\Omega t<200$ are induced by the starting of the electric motor and cannot be avoided in our present experimental set-up. b) Comparison of the measured growth rates $\sigma$ of the induced magnetic field with the theoretical predictions for $\epsilon=0.095$. The error bars have been obtained from the error calculation of the exponential fits of the experimental curves.

limit. An elliptical top was even built in order to illustrate the expected inviscid dynamics. Finally, the induction of a magnetic field by the spin-over has been studied theoretically as well as experimentally. As expected, we have succeeded in recording the exponential growth of a magnetic dipole, induced by the spin-over close to instability threshold. The measured growth rates have been shown to compare well with the hydrodynamics estimates where the Lorenz force is not taken into account. In the experiments, the magnetic Reynolds number was quite limited but we have shown theoretically that the orientation of the induced dipole progressively aligns with the spin-over direction when $R e_{m}$ tends to infinity. This work was initially inspired by the work of Kerswell and Malkus (1998) about the magnetic field of Io, the inner most moon of Jupiter. But we expect that inertial instabilities will also play a major role in other planetary fluid cores as well as in astrophysical flows like accretion disks (Lebovitz and Zweibel, 2004) and binary stars... Therefore, in addition to a more global knowledge of the magnetohydrodynamics of inertial instabilities, we expect that the present studies will contribute to quantify the relative importance of each physical mechanism in the energy balance of such natural flows, and especially in the energy balance of planetary dynamos. 


\section{REFERENCES}

Aldridge, K. D., Seyed-Mahmoud, B., Henderson, G. and Van Wijngaarden, W., Elliptical instability of the earth's fluid core, Phys. Earth Planet. Int., 1997, 103, 365-374.

Anderson, J. D., Sjogren W. D. and Schubert G., Galileo Gravity Results and the Internal Structure of Io, Science, 1996, 272, 709-712.

Busse, F. H., Homogeneous dynamos in planetary cores and in the laboratory, Annu. Rev. Fluid Mech., 2000, 32, 383-408.

Busse, F. H., Convective flows in rapidly rotating spheres and their dynamo action, Phys. Fluids, 2002, 14, 1301-1314.

Eloy, C., Le Gal, P. and Le Dizès, S., Elliptic and triangular instabilities in rotating cylinders, J. Fluid Mech., 2003, 476, 357-388.

Eloy, C. and S., Le Dizès, S. Stability of the Rankine vortex in a multipolar strain field, Phys. Fluids, 2001, 13(3),660-676.

Gledzer, E.B., Dolzhansky, F.V., Obukhov, A.M. and Ponomarev, V.M., An experimental and theoretical study of the stability of motion of a liquid in an elliptical cylinder, Isv. Atmos. Ocean. Phys., 1975, 11, 617-622.

Greenspan, H.P., The Theory of rotating fluids, 1968. Cambridge: Cambridge University Press.

Kerswell, R.R. and Malkus, W.V.R., Tidal instability as the source for Io's magnetic signature, Geophys Res Lett, 1998, 25, 603-606.

Kerswell, R.R., Elliptical instability, Annual Review of Fluid Mechanics, 2002, 34, 83-113.

Kerswell, R.R., Tidal excitation of hydromagnetic waves and their damping in the Earth, J. Fluid Mech., 1994, 274, 219-241.

Kivelson, M. G., Khurana, K. K., Walker, R. J., Russel, C. T., Linker, J. A., Southwood, D. I. and Polanskey, C., A magnetic Signature at Io: Initial Report from the Galileo Magnetometer, Science, 1996, 273, $337-$ 340.

Kivelson, M. G., K. K. Khurana, C. T. Russell, R. J. Walker, J. Warnecke, F. V. Coroniti, C. Polanskey, D. J. Southwood, and G. Schubert, Discovery of Ganymedes magnetic field by the Galileo spacecraft Nature, 1996, 384, 537-541.

Labrosse, S., J.-P. Poirier, and J.-L. Le Moul, The Age of the Inner Core, Earth Planet. Sci. Lett., 2001, 190, 111-123.

Lacaze, L., Le Gal, P. and Le Dizès, S., Elliptical instability in a rotating spheroid, J. Fluid Mech., 2004, 505, $1-22$.

Lacaze, L., Le Gal, P. and Le Dizès, S., Elliptical instability of a flow in a rotating shell, Phys. Earth Planet. Int. , 2005, 151, 194-205.

Lebovitz, N.R. and Zweibel, E., Magnetoelliptic Instabilities, The Astrophysical Journal, 2004, 609, part 1, 301312.

Leweke, T. and Williamson, C. H. K., Cooperative elliptic instability of a vortex pair, J. Fluid Mech., 1998, 360, 85-119.

McEwan, A. D., Inertial oscillations in a rotating fluid cylinder , J. Fluid Mech., 1970, 40, 603-640.

Malkus, W.V.R., An experimental study of global instabilities due to tidal (elliptical) distortion of a rotating elastic cylinder, Geophys. Astrophys. Fluid Dynamics, 1989, 48, 123-134.

Morse, P. M. and Feshbach, H., Methods of theoretical physics, Part I E II, 1953. McGraw-Hill Book Company.

Noir, J., D. Brito, K. Aldridge, and P. Cardin, Experimental evidence of inertial waves in a precessing spheroidal cavity, Geophysical Research Letters, 2001, 19, 3785-3788.

Pierrehumbert, R.T., Universal short-wave instability of two-dimensional eddies in an inviscid fluid, Phys. Rev. Lett., 1986, 57, 2157-2160.

Stevenson, D. J., Planetary magnetic Fields, Earth Planet. Sci. Lett., 2003, 208, 1-11.

Tilgner, A., Precession driven dynamos , Phys. Fluids, 2005, 17, 034104.

Vanyo, J., P. Wilde, Cardin, P., and Olson, P., Experiments on precessing flows in the Earth's liquid core, Geophys. J. Int., 1995, 121, 136-142.

Walleffe, F., On the three-dimensional instability of strained vortices, Phys. Fluids, 1990, 2(1), 76-80.

Wienbruch, U. and Spohn, T., A self-sustained magnetic field on Io?, Planet. Space. Sci., 1995, 43, 1045-1057. 